\section{Polleninformationen kommen jetzt aus Berlin}

Die Geschäftsstelle der Stiftung Deutscher Polleninformationsdienst (PID) hat zum 1. Januar 2010 ihren Sitz von Bad Lippspringe nach Berlin verlegt. Aufgaben und Strukturen der Stiftung gelten auch nach dem Umzug unverändert weiter. Ebenso wird die Arbeit der Messstellen gleichbleibend fortgesetzt.

Die PID-Geschäftsstelle kann in Berlin auf die Unterstützung der Europäischen Stiftung für Allergieforschung (ECARF) und des Allergie-Centrum-Charité bauen. Das Medizinische Zentrum für Gesundheit $\mathrm{GmbH}$ ist dagegen nicht mehr im Beirat der Stiftung vertreten.

Mit dem Umzug ist auch Wechsel in der Betreuung der Geschäftsstelle verbunden. Auf Frau Margarete Wilhelm, die die Geschäftsstelle in Bad Lippspringe 1991 aufgebaut hat, folgt Frau Anja Bode.

Die neuen Kontaktdaten lauten: Stiftung Deutscher Polleninformationsdienst, Geschäftsstelle, Charitéplatz 1, 10117 Berlin, Tel.: (0 30) 4505180 06. Gleich geblieben ist dagegen die Internetadresse www.pollenstiftung.de.

Die Geschäftsstelle ist von Montag bis Donnerstag zwischen 9:30 und 13:30 Uhr und am Freitag von 9:30 bis 13:00 Uhr erreichbar.

\section{Aus BGFA wird IPA}

Das vormalige Berufsgenossenschaftliche Forschungsinstitut für Arbeitsmedizin (BGFA) in Bochum hat seit dem 20. November 2009 einen neuen Namen: Institut für Prävention und Arbeitsmedizin (IPA) der Deutschen Gesetzlichen Unfallversicherung, Institut der Ruhr-Universität, Bochum.

Mit der neuen Bezeichnung soll nicht nur das breite wissenschaftliche Spektrum des Instituts dokumentiert, sondern auch verdeutlicht werden, dass die Arbeit des Instituts allen Mitgliedern der Deutschen Gesetzlichen Unfallversicherung, also den Berufsgenossenschaften und den Unfallkassen gleichermaßen, zugutekommt.

Die Namensänderung ist nicht mit organisatorischen Änderungen verbunden, auch die jeweiligen Ansprechpartner ändern sich nicht. Die neue Internetadresse lautet www.ipa-dguv.de. Unverändert weiter gelten die bisherigen Telefon- und Faxnummern sowie die Hausanschrift. Neu ist dagegen die Postanschrift: IPA, Postfach 102244, 44702 Bochum.

\title{
Kanert-Preis 2009 bleibt in Davos
}

E in Forschungsteam der Hochgebirgsklinik Davos hat im Rahmen des diesjährigen 25. Fortbildungskongresses „Fortschritte der Allergologie, Dermatologie, Pneumologie und Immunologie" in Davos den Förderpreis für Allergieforschung 2009 der KanertStiftung erhalten. Der mit 10.000 Euro dotierte Preis wurde persönlich vom Stifter Horst H. Kanert an Dr. Gundi Willer, Dr. Harald Jung und PD Dr. Günter Menz überreicht.

Mit dem Preisgeld wird ein Forschungsvorhaben unterstützt, das sich mit dem Analgetika-Asthma-Syndrom beschäftigt. Zielsetzung ist, für betroffene Patienten eine Optimierung des Therapiemanagements in Bezug auf Sicherheit und Effektivität zu erzielen.

Von 2004 bis 2006 wurde an der Hochgebirgsklinik Davos in Kooperation mit der HNO-Klinik der LudwigMaximilians-Universität, München, eine adaptive Desaktivierung bei Patienten mit Rezidivpolyposis und Analgetikaintoleranz durchgeführt. Durch diese In- tervention konnten weitere operative Eingriffe im Bereich der Nasennebenhöhlen verhindert und die Lebensqualität verbessert werden. Bei Patienten mit Analgetika-Asthma-Syndrom ist die adaptive Desaktivierung in Kombination mit der operativen Sanierung der Nasennebenhöhlen eine Erfolg versprechende Therapieoption.

Weiter wurde eine Analyse der Daten der Patienten durchgeführt, die von Juli 2007 bis September 2008 in der Hochgebirgsklinik Davos auf eine entsprechende Acetylsalicylsäure-Behandlung eingestellt wurden. Insgesamt wurde diese Therapie bei 35 Patienten durchgeführt. Komplikationen traten bei keinem der Patienten auf.

Insgesamt bestätigen diese Daten die Wirksamkeit und Sicherheit der adaptiven Desaktivierung bei Patienten mit Analgetikaintoleranz und chronischer Pansinusitis. Eine gute Aufklärung der Patienten und Instruktion der weiterbehandelnden Ärzte erhöht die Aussicht auf Erfolg. red

\section{DGAKI hat neue Geschäftsstelle}

$\mathrm{N}$ ach zehn Jahren hervorragender Arbeit in der Geschäftsstelle der Deutschen Gesellschaft für Allergologie und klinische Immunologie hat sich Frau Erika Ratzinger zum Jahresende 2009 in den Ruhestand verabschiedet. DGAKI-Präsident Prof. Dr. Claus Bachert dankte ihr zum Abschied im $\mathrm{Na}$ men des Vorstandes für die immer kompetente und freundliche Mitarbeit!

Die Leitung der Geschäftsstelle hat zum 1. Januar neu Frau Gertrud Hammel übernommen. Mit dem Wechsel in der personellen Betreuung ist auch ein Ortswechsel der Geschäftsstelle von München nach Aystetten, westlich von Augsburg, verbunden. Die neuen Kontaktdaten sind im Kasten rechts zusammengefasst. Der Vorstand der DGAKI und auch die Redaktion des Allergo Journal wünschen Frau Hammel einen guten Start!

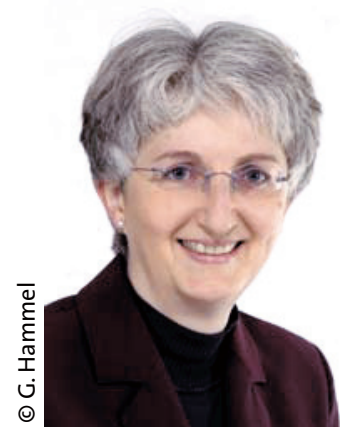

Leitet seit 1. Januar die Geschäftsstelle der DGAKI: Gertrud Hammel
Neue Kontaktdaten Geschäftsstelle

Geschäftsstelle DGAKI

Postfach 05

86482 Aystetten

Tel.: (08 21) 48687864

Fax: (08 21) 48687863

E-Mail:info@dgaki.de 